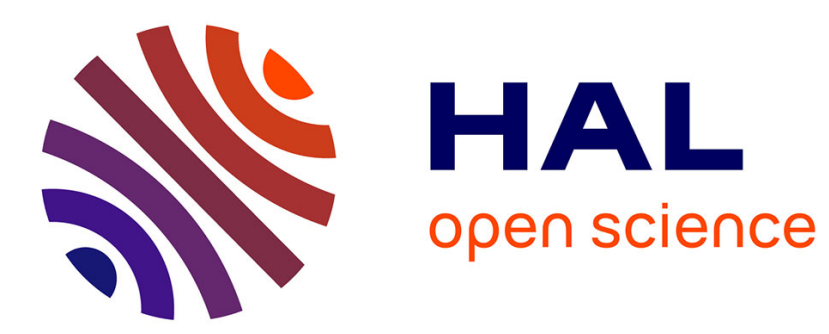

\title{
Hybrid prognostic method applied to mechatronic systems.
}

Kamal Medjaher, Noureddine Zerhouni

\section{To cite this version:}

Kamal Medjaher, Noureddine Zerhouni. Hybrid prognostic method applied to mechatronic systems.. International Journal of Advanced Manufacturing Technology, 2013, pp.1-20. 10.1007/s00170-0135064-0 . hal-00867575

\section{HAL Id: hal-00867575 https://hal.science/hal-00867575}

Submitted on 30 Sep 2013

HAL is a multi-disciplinary open access archive for the deposit and dissemination of scientific research documents, whether they are published or not. The documents may come from teaching and research institutions in France or abroad, or from public or private research centers.
L'archive ouverte pluridisciplinaire HAL, est destinée au dépôt et à la diffusion de documents scientifiques de niveau recherche, publiés ou non, émanant des établissements d'enseignement et de recherche français ou étrangers, des laboratoires publics ou privés. 


\title{
Hybrid Prognostic Method Applied to Mechatronic Systems
}

\author{
K. Medjaher · N. Zerhouni
}

Received: date / Accepted: date

\begin{abstract}
Fault detection and isolation, or fault diagnostic, of mechatronic systems has been subject of several interesting works. Detecting and isolating faults may be convenient for some applications where the fault does not have severe consequences on humans as well as on the environment. However, in some situations, diagnosing faults may not be sufficient and one needs to anticipate the fault. This is what is done by fault prognostics. This latter activity aims at estimating the remaining useful life of systems by using three main approaches: data-driven prognostics, model-based prognostics and hybrid prognostics. In this paper, a hybrid prognostic method is proposed and applied on a mechatronic system. The method relies on two phases: an offline phase to build the behavior and degradation models and an online phase to assess the health state of the system and predict its remaining useful life.
\end{abstract}

Keywords Fault Detection · Fault Diagnostics · Fault Prognostics · Remaining Useful Life · Bond Graph Modeling.

\section{Introduction}

Fault Detection and Isolation (FDI), and fault prognostics of industrial systems are two necessary functions as they allow avoiding non-desirable situations and catastrophes. FDI can be applied on both abrupt and incipient faults. Several research and industrial works have been conducted in the domain $[6,7,16,19]$. The reported methods can be classified in two main categories: qualitative methods and quantitative methods $[6,16]$. FDI can be used to do reconfiguration and accommodation and is suitable for systems where

K. Medjaher and N. Zerhouni

FEMTO-ST Institute, UMR CNRS 6174 - UFC / ENSMM / UTBM

Automatic Control and Micro-Mechatronic Systems Department

24, rue Alain Savary, 25000 Besançon, France

E-mail: kamal.medjaher@ens2m.fr 
the fault does not have severe consequences. For example, detecting and isolating a fault on a valve controlling inflammable liquids may not avoid possible explosions. In this case, the fault is diagnosed a posteriori and thus is undergone.

Contrary to FDI, which is done a posteriori after the appearance of the faults, prognostics aims at anticipating the time of a failure by predicting the Remaining Useful Life (RUL) of the system [1]. Prognostic results can then be used to take appropriate decisions on the system (change of set points, reduce the production load, stop the system, etc.).

Fault prognostic methods can be grouped in three main approaches $[5,7,17$, 18]: data-driven prognostics, model-based prognostics and hybrid prognostics. Data-driven prognostics is based on the utilization of monitoring data to build behavior models including the degradation evolution, which are then used to predict the RUL [3,4]. Model-based prognostics, also called physics of failure prognostics, uses models generated from fundamental laws of physics to calculate the RUL $[2,11]$. Finally, hybrid prognostics combines both previous approaches and benefits from their advantages (precision and applicability).

This paper presents a hybrid prognostic method with application to mechatronic systems. In this contribution, the behavior model is obtained by using the Bond Graph (BG) formalism $[8,16]$ and the degradation models are derived by using the concept of residuals. The degradation of the system's components are supposed to be continuous drifts in the system's parameters. The global model of the mechatronic system (behavior and degradation models) is then used to estimate the current health state of the system, predict its future one and calculate its RUL.

The paper is organized as follows. After the introduction, section 2 presents a brief description of the bond graph formalism and the fault prognostic paradigm. Section 3 gives the framework and details the steps of proposed method. Section 4 deals with the application of the method on a mechatronic system, where simulation results are presented and discussed. Finally, section 5 concludes the paper.

\section{Bond graph and fault prognostics}

\subsection{Bond graph modeling}

Bond graph tool $[8,16]$ is a graphical representation of power transfer within a physical system. A bond graph model is situated between the physical model and the mathematical model. It is used in modeling to derive mathematical models in forms of state space and transfer function, in structural analysis of the system's properties like controllability, observability, model reduction, actuator and sensor placement, and finally in fault detection and isolation. The generation of a BG model is based on nine BG elements: three passive elements (resistance $R$, capacitance $C$ and inertia $I$ ), two active elements (source of effort $S e$ and source of flow $S f$ ) and four junction elements (transformer 

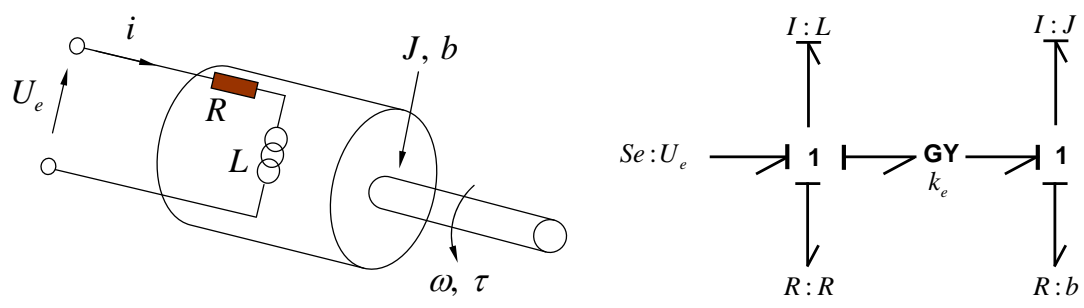

Fig. 1: A DC motor and its BG model.

$T F$, gyrator $G Y$, zero junction 0 and one junction 1 ). In addition to these nine elements, two detectors representing the sensors are added (effort and flow detectors). These BG elements are proposed to unify the modeling process of multi-physical systems, by using two generalized variables: effort and flow. The product of these two variables is equal to power, which is exchanged between the physical parts of the system.

To obtain a BG model of multi-physical systems in general and of mechatronic systems in particular, it is recommended to start with a word BG. This word BG is obtained by decomposing the whole system into several energydomain parts (electrical, mechanical, hydraulic, pneumatic, thermal, etc.) and linking these parts between them by using half-arrows representing the power exchanged between them and called bond graph links. Then, for each block a BG model is derived by using dedicated procedures given in [8]. A BG model of a DC motor, composed of two coupled physical domains (electrical and mechanical) is given in figure 1 .

\subsection{Fault prognostics}

Prognostics is a key process of Condition-Based Maintenance (CBM) $[9,15]$ (figure 2). Prognostics is defined by the international standard organization [1] as the estimation of the operating time before failure and the risk of future existence or appearance of one or several failure modes. The time to failure is commonly called remaining useful life (RUL) by the Prognostics and Health Management (PHM) research community [5,7,12,18]. Figure 3 shows an illustration of a RUL prediction according to a predefined system's performance. Fault prognostics can be done according to three main approaches: data-driven prognostics, model-based (also called physics of failure) prognostics and hybrid prognostics. The first approach uses the data provided by sensors (monitoring data) and which capture the degradation evolution of the system. The data are then preprocessed to extract features which are used to learn models for health assessment and RUL prediction [3,4]. Examples of models are neural networks, regressions, hidden Markov models, support vector regression, etc. The second approach requires a deep understanding of the physical phenomena of the system, including the degradation evolution. This approach uses 


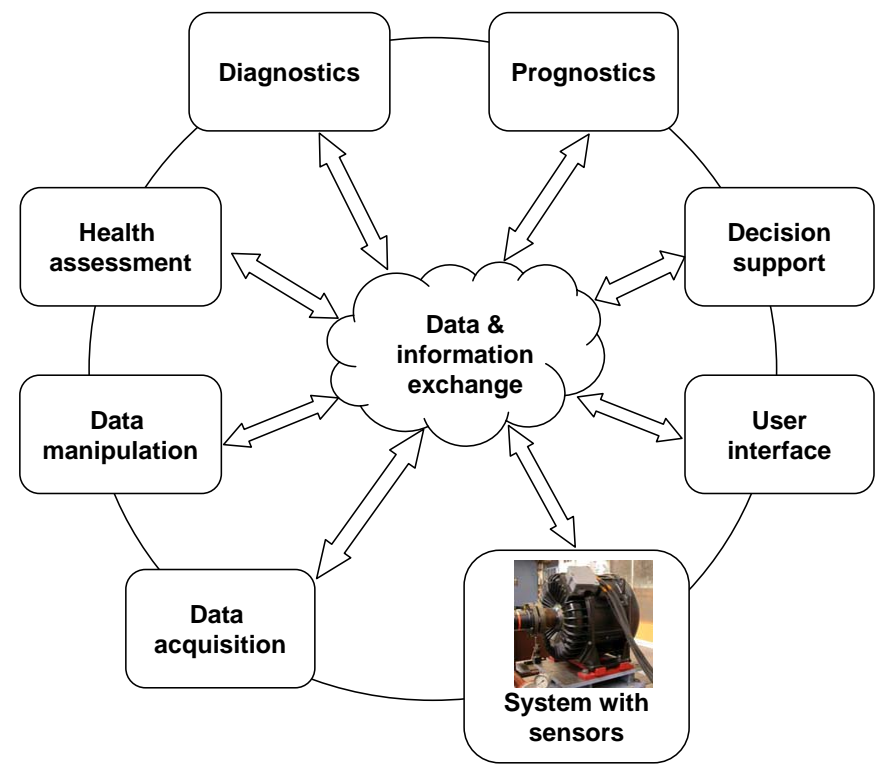

Fig. 2: Steps of a Condition-Based Maintenance.

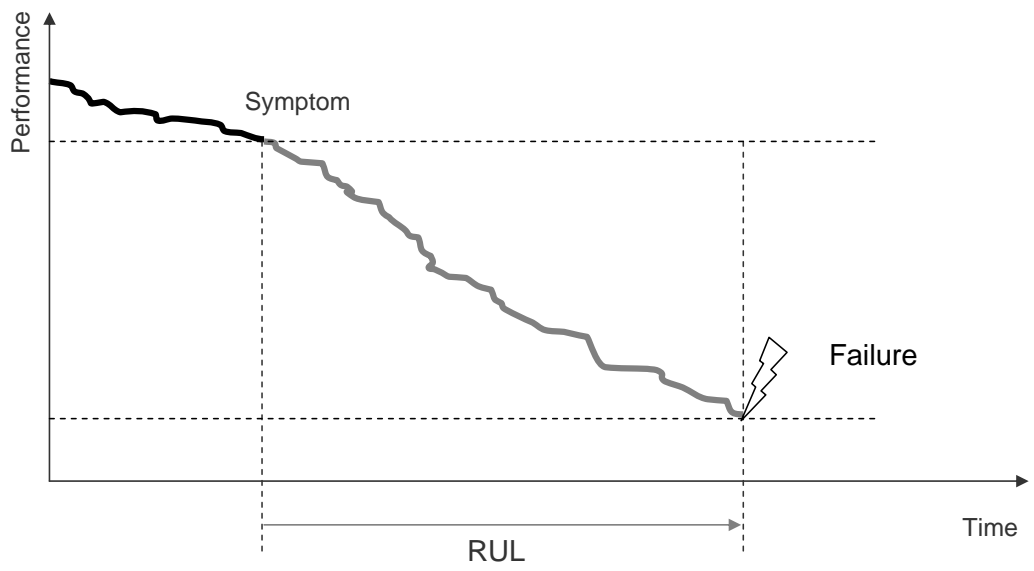

Fig. 3: Illustration of a RUL prediction.

physical laws to build the global model of the system, which is then used for simulations and predictions to calculate the RUL $[2,11]$. Note that the construction of the model is subjected to the availability of a degradation model. Examples of degradation models are those related to crack by fatigue, corrosion and wear. Finally, the third approach combines both previous approaches. The advantage of the hybrid approach is that it allows doing reliable prognostics at two levels: component level prognostic and system level prognostic. The 
component level prognostic allows building accurate degradation models which can then be injected in the global model obtained at the system level in order to estimate the remaining useful life of the whole system. Furthermore, the hybrid approach allows modeling the interactions between the components of the system and thus tracking the influence of a degradation in one component on the other components.

A summary of the advantages and drawbacks of the previous approaches is given in figure 4 . Compared to the model-based approach, data-driven methods
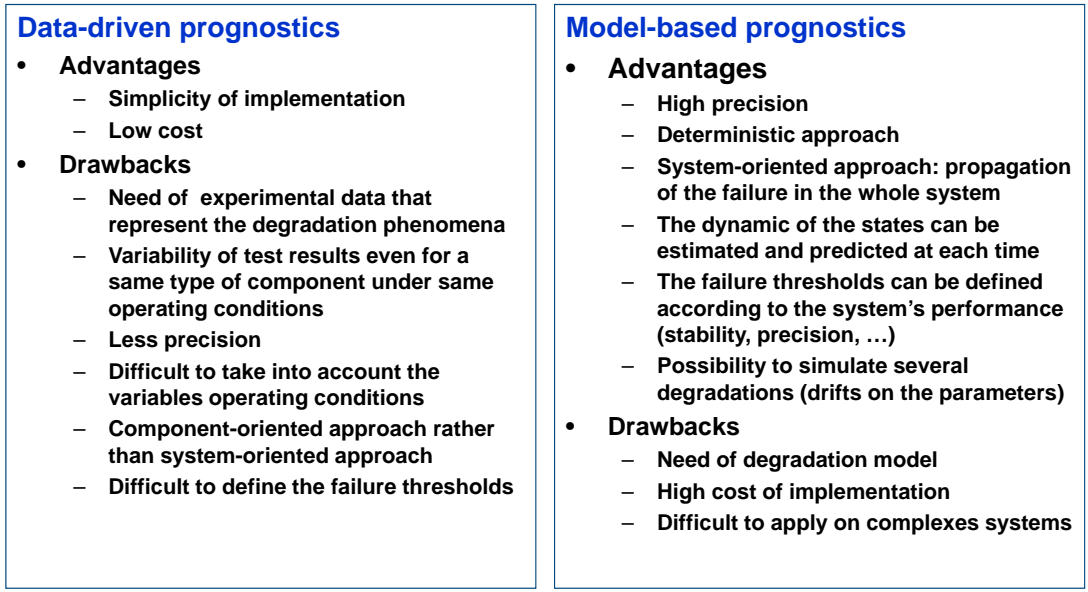

Fig. 4: Data-driven prognostics vs Model-based prognostics.

give less precise prognostics $[10,18]$, due particularly to the absence of a deterministic behavior model and to the variability of the experimental data needed to learn the degradation model of the physical system. The model-based methods give more precise results, but their implementation on complex physical systems is not trivial because of the difficulty to generate the system's behavior and degradation models. However, these methods can be applied on small system for which the behavior model can be easily obtained. This is the case of mechatronic systems. Nevertheless, even for these systems it is necessary to have the models of the degradation phenomena before doing prognostics. The degradation models can be learned from experimental data acquired on accelerated life tests done on the system's components or estimated online by using appropriate techniques (residuals, parameter estimation, observers, etc.). Once the degradation models of the components are obtained, they are injected in the behavior model of the system to estimate and predict its health state and calculate its RUL.

The following of the paper presents a prognostic method applied on mechatronic systems. The method combines both model-based and data-driven approaches. The behavior model of the system is obtained by using the BG tool 
whereas the degradation models of the system's components are derived by using the concept of residuals.

\section{Fault prognostics of mechatronic systems}

The prognostic method proposed in this paper relies on two phases, as shown in figure 5: an offline phase to build the dynamic model of the mechatronic system and derive its degradation models, and an online phase (or exploitation phase) where the obtained models are used to detect the initiation of the degradation and predict the RUL of the system. Note that, contrary to most reported prognostic works which are component-oriented, the method proposed in this paper is system-oriented. Indeed, the variations (or drifts) in the parameters are propagated to the whole mechatronic system and are taken into account in the global dynamic model for simulations, predictions and RUL calculation.

Before detailing the steps of each phase shown in figure 5 , it is necessary to

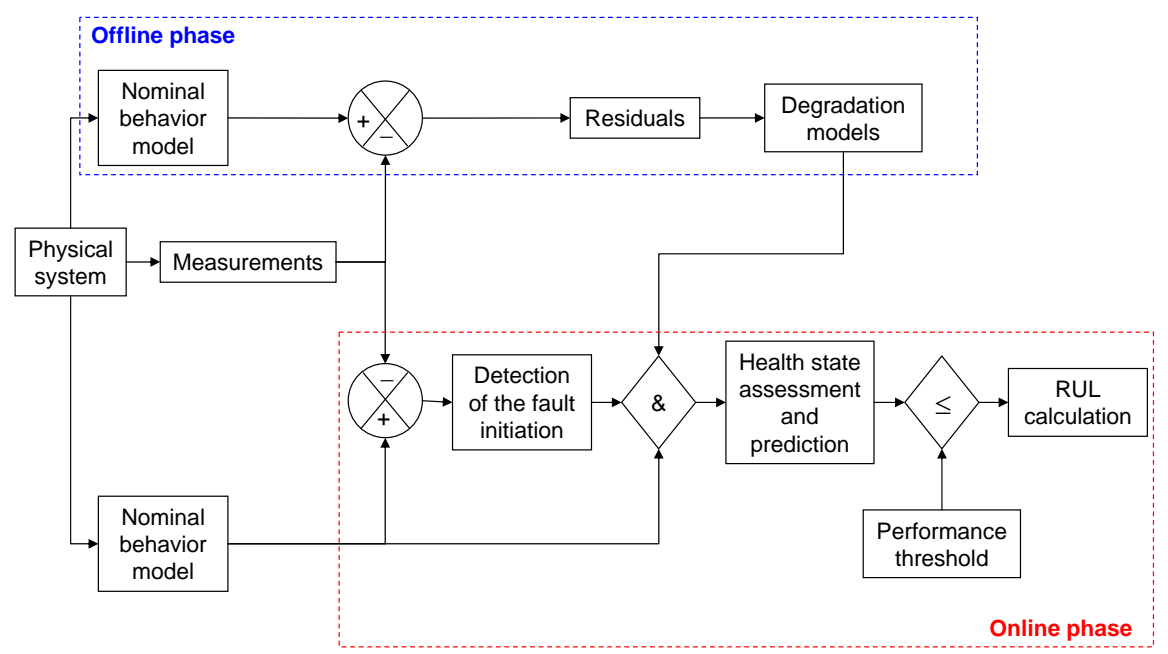

Fig. 5: Overview of the proposed prognostic method.

set the framework of the proposed method. This framework is defined by the following assumptions.

1. The sensors are considered to be fault free and give correct measurements.

2. Only incipient faults are considered (abrupt faults are not taken into account).

3. The faults in the mechatronic system are due to continuous drifts in its parameters.

4. The faults in the actuators are not taken into account. 
The first phase of the method includes three steps: the construction of the nominal behavior model of the system, the generation of its degradation model and the definition of the thresholds (faults' thresholds and system's performance thresholds). The nominal model consists of a set of mathematical equations obtained by using the bond graph formalism $[8,16]$. The output of this model is compared to the measurements acquired on the real system to generate residuals, which are then used to derive the degradation models of the system's components. The degradations correspond to changes in the BG elements $C$, $I$ and $R$, as expressed by the following relations.

$$
\begin{gathered}
C(t)=C_{0}+f(t) \\
I(t)=I_{0}+g(t) \\
R(t)=R_{0}+h(t)
\end{gathered}
$$

$C_{0}, I_{0}$ and $R_{0}$ are the nominal values of the BG elements $C, I$ and $R$, respectively and $f(t), g(t)$ and $h(t)$ are the time variations of these elements. For example, a degradation in the stator of an electrical machine can be interpreted as a continuous modification of the electrical resistance of the stator winding. Similarly, a degradation of battery can be explained by a modification of its electrical capacitance.

A residual is a numerical evaluation of an Analytical Redundancy Relations (ARR) obtained from an over-determined system of equations (number of equations is greater than the number of variables) [13,14]. An ARR is obtained from an over-determined system of equations (number of equations is greater than the number of variables). An ARR contains only known variables (inputs, outputs and parameters of the system) and it is represented by the following expression.

$$
\operatorname{ARR}: \Phi(K)=0,
$$

where $K$ is the set of known variables. An ARR can represent mass balance, energy balance, etc.

A residual $r(t)$ is a numerical evaluation of an ARR.

$$
r(t)=\Phi(K)
$$

The residual are signal which are used to verify the coherence between the nominal and the actual behavior of the system. When the system operates correctly, the residuals' values should be theoretically equal to zero; otherwise, the residuals increase (or decrease) as the system leaves its nominal behavior. Figure 6 shows the principle of a residual signal. In this contribution, the ARRs and the corresponding residuals are obtained from the BG model by applying the following procedure (more details can be found in $[13,14]$ ).

1. Build the bond graph model in preferred integral causality of the mechatronic system.

2. Put the bond graph model in preferred derivative causality (with inversion of the sensors' causality if necessary). 


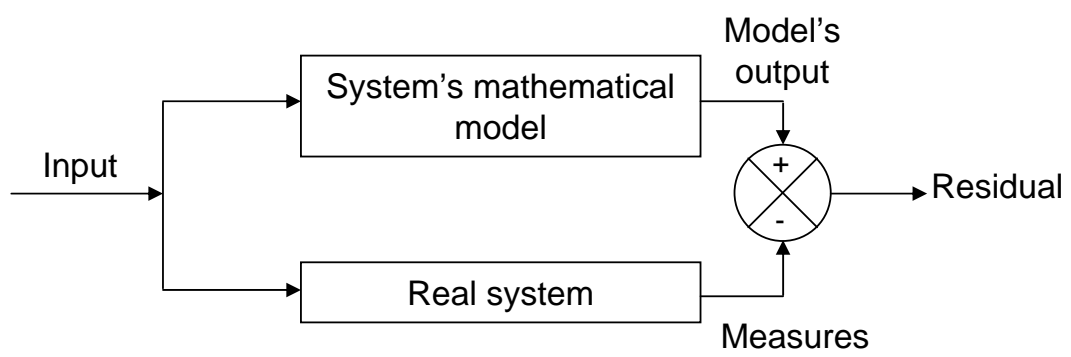

Fig. 6: Principle of residuals.

3. Write the constitutive relation for each junction of the bond graph model in preferred derivative causality.

4. Eliminate the unknown variables from each constitutive relation by covering the causal paths on the bond graph model.

Let $\alpha_{1}, \alpha_{2}, \ldots \alpha_{n}$ be the set of physical parameters of the system which are involved in its dynamic model and in the corresponding residuals. The residual equation given in equation (5) can then be re-written as follows:

$$
r(t)=\Phi\left(\alpha_{1}, \alpha_{2}, \alpha_{3}, \ldots \alpha_{n}\right)
$$

Then, the evolution of the degradation can be determined by inverting the equation (6). For example, in the case where the degradation corresponds to the variation of the parameter $\alpha_{1}$, its evolution can be calculated by the following equation.

$$
\alpha_{1}=\Phi^{-1}\left(r(t), \alpha_{2}, \alpha_{3}, \ldots \alpha_{n}\right)
$$

The second phase of the proposed method concerns the exploitation of the models and knowledge obtained in the first phase to assess the health state of the system and calculate its RUL. During this phase, the output of the nominal behavior of the system is continuously compared to the measurements provided by the sensors to detect whether the fault starts to occur or not. If a fault initiation is detected, the process of health assessment and RUL calculation is launched. The detection of a fault initiation is done by continuously evaluating the residuals and by analyzing the corresponding binary fault signature matrix formed by the residuals.

The global model, composed by the nominal model, the degradation model and the result of the fault detection, is used to assess the health state of the mechatronic system, predict its future one and calculate its RUL. The RUL is calculated according to a defined performance (which can be related to the precision of the system, its stability, etc.) and by using the equation (8), which is illustrated by the figure 7 .

$$
R U L(t)=t_{f}-t_{0}
$$




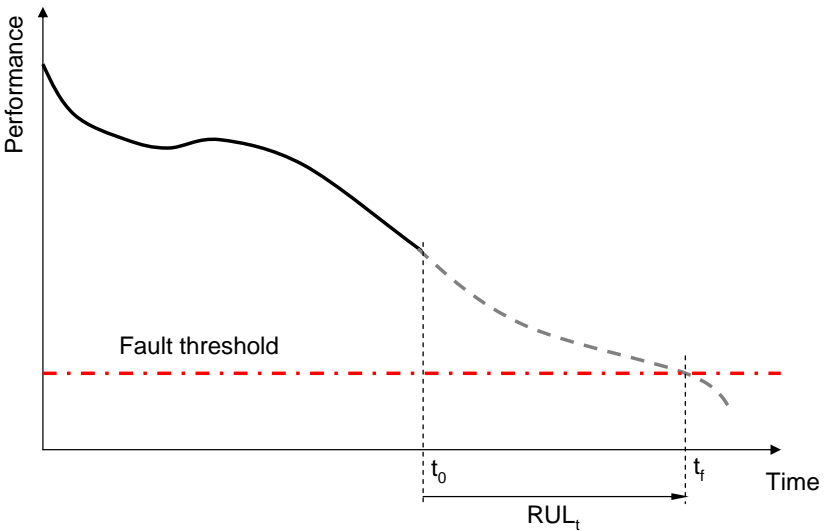

Fig. 7: RUL calculation according to a given performance.

Note: during the exploitation of the mechatronic system, several fault can occur. In the case where the faults occur a the same time, the RUL of the system can be calculated from the individual RULs of the components which are failing. The RUL corresponds then to the shortest RUL among the individual RULs.

\section{Case study and simulation results}

\subsection{Description of the mechatronic system}

The mechatronic system considered for the application of the prognostic method described above is shown in figure 8. The main purpose of this system is to

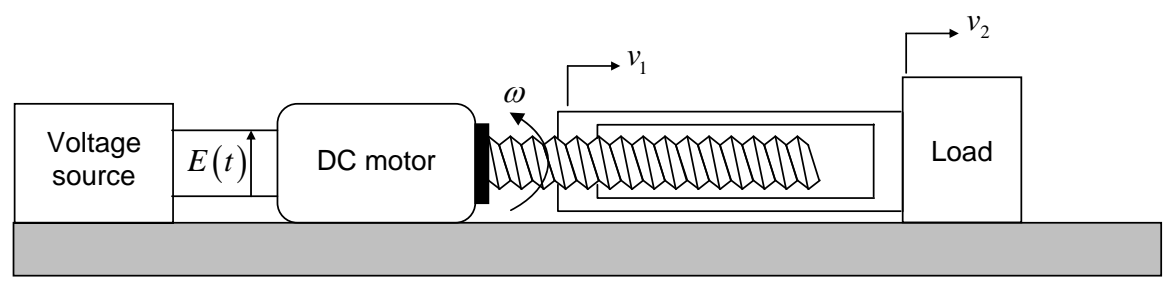

Fig. 8: Scheme of the mechatronic system.

position horizontally a load which is situated at the right side of the scheme [8]. The system is composed of a voltage source, which can be a battery, a DC motor providing a rotational movement and a screw transforming this latter movement to a translational one in order to position horizontally the load. 


\subsection{Behavior model}

The BG model of the system is built by taking into account the following hypotheses.

- The voltage source is constant.

- The electrical part (stator winding) of the DC motor is composed of a resistance and an inductance. Its mechanical part is represented by an inertia and a mechanical friction.

- The translation velocity $v_{1}$ is proportional to the rotation velocity $\omega: \omega=$ $S . v_{1}$.

- The part linking the screw to the mass is not completely rigid and presents some elasticity represented by a stiffness $k$.

- The load has a mass $M$ and is in friction with the support.

The BG model in integral causality of the mechatronic system presented in figure 8 is given in figure 9 .

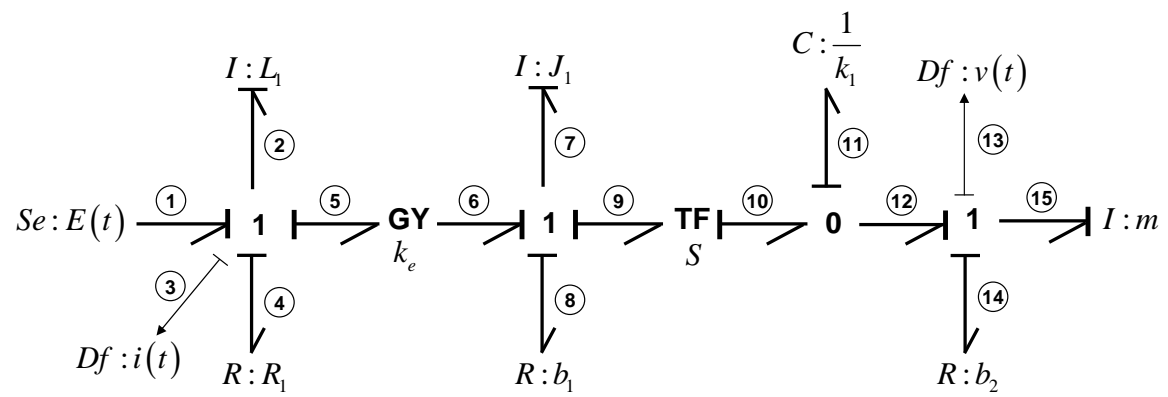

Fig. 9: BG model of the mechatronic system.

The dynamic model of the mechatronic system can be obtained from the BG model in integral causality by following the steps given below.

- Define the inputs, the outputs and the state space variables.

- Write the BG equations (junction equations, TF and GY equations, and constitutive elements' equations). These equations must be written by taking into account the causality of the model.

- Combine the above equations to get the differential form of the state space variables and the output variables as functions of state space and input variables.

The equations derived from the junctions "0", "1", the transformer "TF" and the gyrator "GY" of the bond graph model are given below.

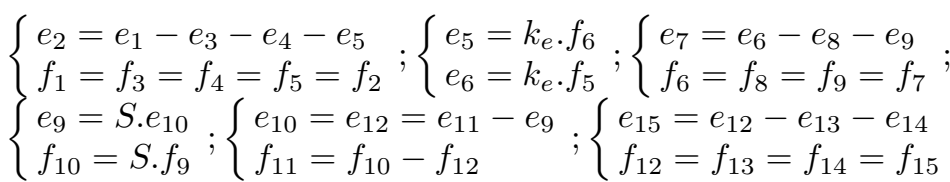




\begin{tabular}{|c|c|c|}
\hline Symbol & Description & Numerical value \\
\hline$E(t)$ & Voltage source & $10 \mathrm{~V}$ \\
\hline$k_{e}$ & Torque coefficient of the DC motor & 0.47 N.m/A \\
\hline$S$ & $\begin{array}{l}\text { Coefficient linking the rotation and translation veloc- } \\
\text { ities }\end{array}$ & $0.01 \mathrm{~m}$ \\
\hline$R_{1}$ & Electrical resistance of the DC motor & $0.61 \Omega$ \\
\hline$L_{1}$ & Inductance of the DC motor & $0.0019 H$ \\
\hline$J_{1}$ & Inertia of the rotation part & 0.01 N.m.s ${ }^{2}$ \\
\hline$b_{1}$ & $\begin{array}{l}\text { Friction coefficient of the mechanical part of the DC } \\
\text { motor }\end{array}$ & $0.3 \mathrm{~N} . \mathrm{s} / \mathrm{m}$ \\
\hline$k_{1}$ & $\begin{array}{l}\text { Stiffness of the linking part between the screw and the } \\
\text { mass }\end{array}$ & $3.33 \times 10^{-6} \mathrm{~N} / \mathrm{m}$ \\
\hline$m$ & Mass & $800 \mathrm{~kg}$ \\
\hline$b_{2}$ & Friction coefficient of the mass $m$ & 3000 N.s $/ m$ \\
\hline$\alpha$ & Predefined parameter related to the degradation & 0.01 \\
\hline
\end{tabular}

Table 1: Values of the parameters used for simulation.

The constitutive equations of the bond graph elements of figure 9 are given in equation (10).

$$
\begin{aligned}
& f_{2}=\frac{1}{L_{1}} \cdot \int e_{2} \cdot d t ; \quad e_{4}=R_{1} \cdot f_{4} ; \quad f_{7}=\frac{1}{J_{1}} \cdot \int e_{7} \cdot d t ; e_{8}=b_{1} \cdot f_{8} \\
& e_{11}=k_{1} \cdot \int f_{11} \cdot d t ; \quad e_{14}=b_{2} \cdot f_{14} ; \quad f_{15}=\frac{1}{m} \cdot \int e_{15} \cdot d t
\end{aligned}
$$

From equations (9) and (10), the dynamic model of the mechatronic system can be obtained in the form of state space (equation (12)). In this equation, the dimension of the state vector $x$ is equal to four.

$$
x=\left(\begin{array}{llll}
p_{2} & p_{7} & q_{11} & p_{15}
\end{array}\right)^{T} \Rightarrow \dot{x}=\left(\begin{array}{llll}
\dot{p}_{2} & \dot{p}_{7} & \dot{q}_{11} & \dot{p}_{15}
\end{array}\right)^{T}
$$

The variable $p_{2}$ stands for the electrical flux of the inductance $L_{1}, p_{7}$ is the momentum of the inertia $J_{1}, q_{11}$ is the displacement of the part linking the screw to the mass and $p_{15}$ is the momentum of the mass $m$. The variable $y$ stands for the output of the mechatronic system and corresponds to the velocity of the mass $m$.

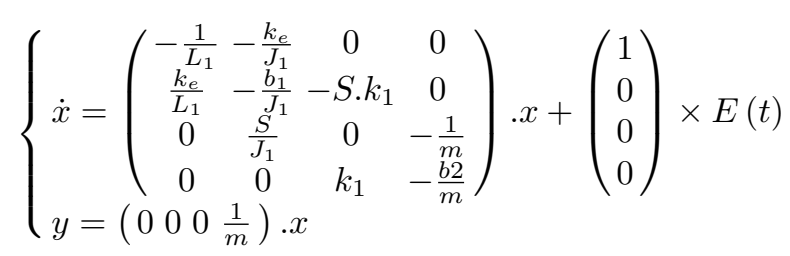

In the following of the section, Matlab ${ }^{\circledR}$ and Simulink ${ }^{\circledR}$ software tools are used to code and simulate the state space equations including the degradation models, and to calculate the remaining useful life of the mechatronic system. The time response of the system to a step input is shown in figure 10. From this figure, we can see that the system is stable and its final value corresponding to the mass velocity (without any degradation taken into account) is equal to $0.08 \mathrm{~m} / \mathrm{s}$. The stability of the system can be easily verified by calculating the eigenvalues of the state matrix given in equation (12). By considering the 
numerical values of the parameters given in table 1 , the eigenvalues of the system are equal to: $-275.74,-033.18+46.99 i,-033.18-46.99 i$ and -12.71 . In the following study, the performance measure according to which the prog-

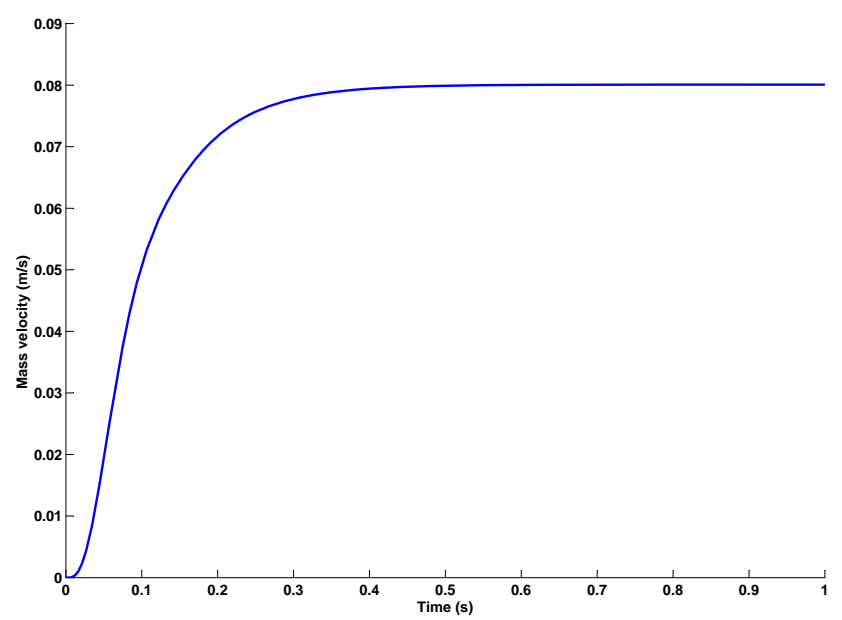

Fig. 10: Step response of the mechatronic system.

nostics is done can be the final value of the response, the stability of the system, the precision, etc. This performance will determine the failure threshold and thus the estimation of the RUL of the system. For example, if the stability is considered, the estimation of the RUL will correspond to the time difference between the current time and the limit time for which the system becomes instable. However, in the case where the stability holds despite the degradation, another measure will be considered (for example an acceptable value of the system's response).

\subsection{Generation of the residuals}

It is necessary to identify the system's components which are subject to degradations and define their degradation models before generating the residuals. In this application, the degradation phenomena which can be taken into account are the drift in the resistance of the DC motor's winding, the magnetic deterioration of the DC motor's permanent magnet and the bending of the rotating shaft. The degradation of the electrical resistance can be caused by the variation of the resistivity of the winding due to temperature change inside the DC motor. The magnetic degradation concerns the diminution of the magnetic field generated by the permanent magnet of the DC motor. Finally, the bending of the shaft can be induced by overloading the DC motor and by external perturbations. 
The ARRs and the corresponding residuals of the mechatronic system shown in figure 8 are obtained by using the steps presented in section 3. By applying these steps, the bond graph model in derivative causality corresponding to the bond graph model given in figure 9 is shown in figure 11. Two ARRs can be

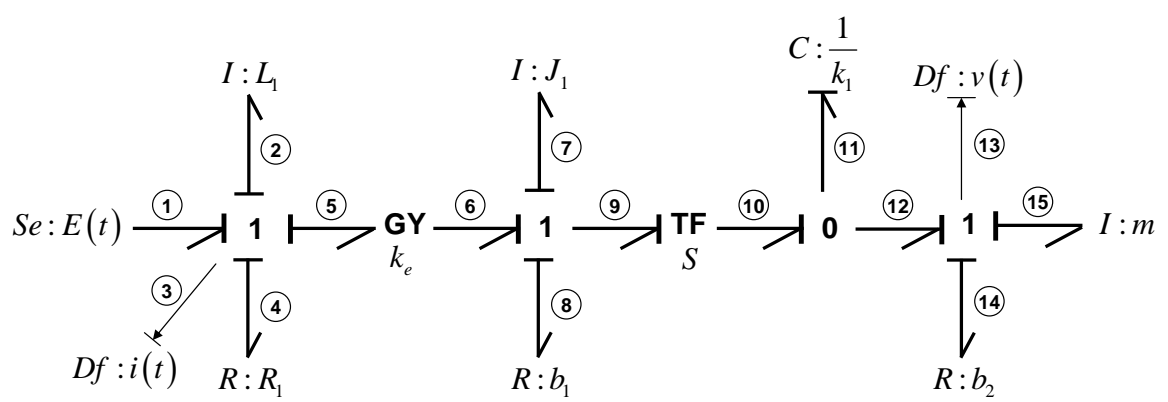

Fig. 11: BG model in derivative causality of the mechatronic system.

derived from the bond graph model in derivative causality (one ARR for each sensor). The first ARR is obtained from the junction equation "1" connected to the flow detector $D f: i(t)$.

$$
e_{3}=e_{1}-e_{2}-e_{4}-e_{5} \Leftrightarrow e_{1}-e_{2}-e_{4}-e_{5}=0
$$

By replacing the unknown variables $e_{1}, e_{2}, e_{4}$ and $e_{5}$ by known variables, the following first ARR can be derived.

$A R R_{1}: E(t)-L_{1} \cdot \frac{d i(t)}{d t}-R_{1} \cdot i(t)-\frac{k_{e}}{S} \cdot\left(v(t)+\frac{b_{2}}{k_{1}} \cdot \frac{d v(t)}{d t}+\frac{m}{k_{1}} \frac{d^{2} v(t)}{d t^{2}}\right)=0$

The second ARR is obtained from the junction equation " 1 " connected to the flow detector $D f: v(t)$.

$$
e_{13}=e_{12}-e_{14}-e_{15} \Leftrightarrow e_{12}-e_{14}-e_{15}=0
$$

By using the same substitutions than for the $\mathrm{ARR}_{1}$, the second $\mathrm{ARR}$ is given below.

$$
\begin{aligned}
& A R R_{2}: k_{e} \cdot i(t)-\frac{b_{1}}{k_{e}} \cdot\left(E(t)-L_{1} \cdot \frac{d i(t)}{d t}-R_{1} \cdot i(t)\right) \\
& -\frac{J_{1}}{k_{e}} \cdot \frac{d}{d t}\left(E(t)-L_{1} \cdot \frac{d i(t)}{d t}-R_{1} \cdot i(t)\right)-b_{2} \cdot S \cdot v(t)-m . S \cdot \frac{d v(t)}{d t}=0
\end{aligned}
$$

The corresponding residuals can be obtained by numerically evaluating the ARRs given in equations (14) and (16). The two obtained residuals are given by the following expressions.

$$
\left\{\begin{array}{l}
r_{1}(t)=E(t)-L_{1} \cdot \frac{d i(t)}{d t}-R_{1} \cdot i(t)-\frac{k_{e}}{S} \cdot\left(v(t)+\frac{b_{2}}{k_{1}} \cdot \frac{d v(t)}{d t}+\frac{m}{k_{1}} \frac{d^{2} v(t)}{d t^{2}}\right) \\
r_{2}(t)=k_{e} \cdot i(t)-\frac{b_{1}}{k_{e}} \cdot\left(E(t)-L_{1} \cdot \frac{d i(t)}{d t}-R_{1} \cdot i(t)\right) \\
-\frac{J_{1}}{k_{e}} \cdot \frac{d}{d t}\left(E(t)-L_{1} \cdot \frac{d i(t)}{d t}-R_{1} \cdot i(t)\right)-b_{2} \cdot S \cdot v(t)-m . S \cdot \frac{d v(t)}{d t}
\end{array}\right.
$$


The advantage of these residuals is that they can be used for fault detection, fault diagnostics and fault prognostics. In the case of fault detection and diagnostics, the residuals are used as balance equations and they do not need the degradation model (which corresponds to the deviation of the winding's resistance in this case study). Indeed, any change in one or more parameters of the mechatronic system will lead to a variation of the residuals in which the parameters are involved. The relationships between the parameters of the mechatronic system and the residuals can be expressed by a fault signature matrix (more details about the construction of this matrix can be found in $[13,14,16])$. This matrix is a binary one, each cell "i" of the matrix contains a value equal to " 1 " if the parameters of a component is present in the residual " $r_{i}$ " and a value equal to "0" otherwise. The "1" values mean that the variation of the parameters of a component will induce a variation of the residuals in which these parameters are present. The analysis of this matrix allows to clearly determine which component (or group of components) is faulty.

In the case of fault prognostics, the integration of the degradation should be taken into account in the global model of the system to predict its RUL. In this contribution, the residuals are used to detect the initiation of the degradation on the mechatronic system. For example, in the case of a degradation on the electrical resistance of the motor's winding, the values of both residuals $r_{1}$ and $r_{2}$ will change. This is because the parameter $R_{1}$ related to the electrical resistance is present in the two residuals. The degradation model of the resistance can then be obtained by inverting the residuals' equations. By using the residual $r_{1}$, the variation of the resistance can be expressed by the following equation.

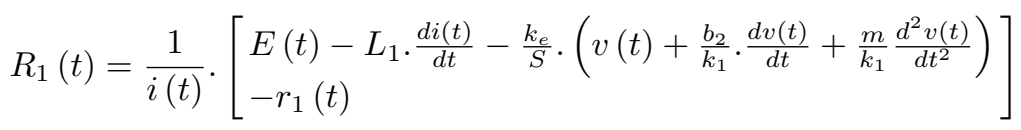

In practice, the degradation models of the system's components can be obtained offline by realizing accelerated life experiments. Then, the derived models are integrated to the behavior model of the system, and the whole model is exploited to assess the system's health state and predict its RUL. The trend of the degradation extracted from equation (18) is shown in figure 12. From this figure, one can observe a linear degradation, which can be expressed by the following formula.

$$
R(t)=R_{1} \cdot(1+\alpha . t)
$$

where $R_{1}$ is the nominal value of the resistance (absence of degradation). Indeed, the electrical resistance of the winding can be expressed by the following equation:

$$
R=\rho \cdot \frac{L}{S}
$$

where $\rho$ is the resistivity of the winding, $L$ its length and $S$ its cross section. The degradation of the resistance $R_{1}$ is due mainly to the degradation of its resistivity $\rho$ (the variation of the length $L$ is compensated by the variation of 


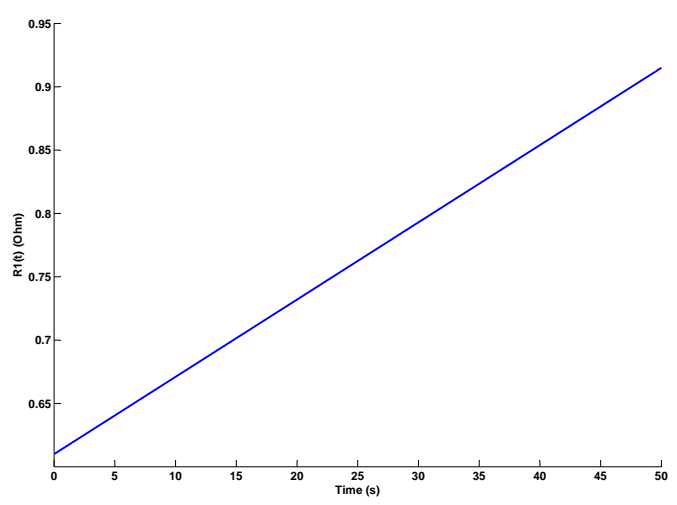

Fig. 12: The linear trend of the degradation.

the cross section $S$ ). The variation of the resistivity can be expressed by the following equation:

$$
\rho(t)=\rho_{0} \cdot(1+\alpha . t)
$$

where $\rho_{0}$ is the nominal value of the resistivity. Thus, $R(t)=R_{1} \cdot(1+\alpha . t)$, where $R_{1}=\rho_{0} \cdot \frac{L}{S}$.

In addition to the linear model of the resistance degradation given in equation (19), a nonlinear degradation model, with $R(t)=R_{1} \cdot \mathrm{e}^{0.1 . t}$, is simulated (figure 13). The results of the simulations related to linear and nonlinear degradations are given in the following subsection.

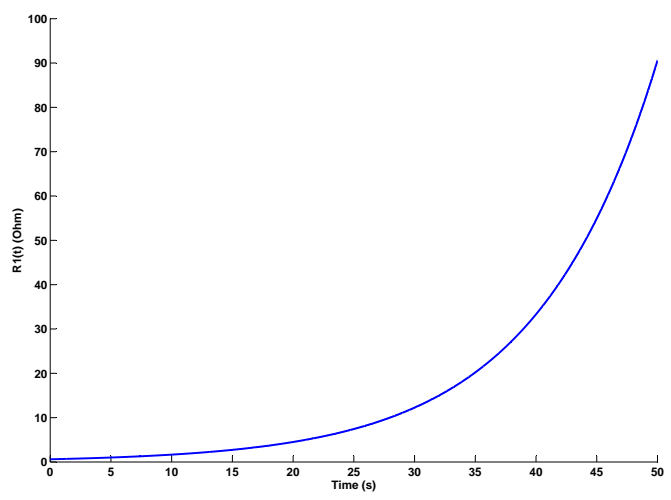

Fig. 13: A nonlinear degradation model. 


\subsection{RUL estimation}

In the absence of failures, the two residuals generated previously should have mean values close to zero. In this case, the residuals are conservative (the algebraic sum of the applied forces on the mechatronic system is equal to zero). The figure 14 shows the time evolution of the residuals. However, in the pres-
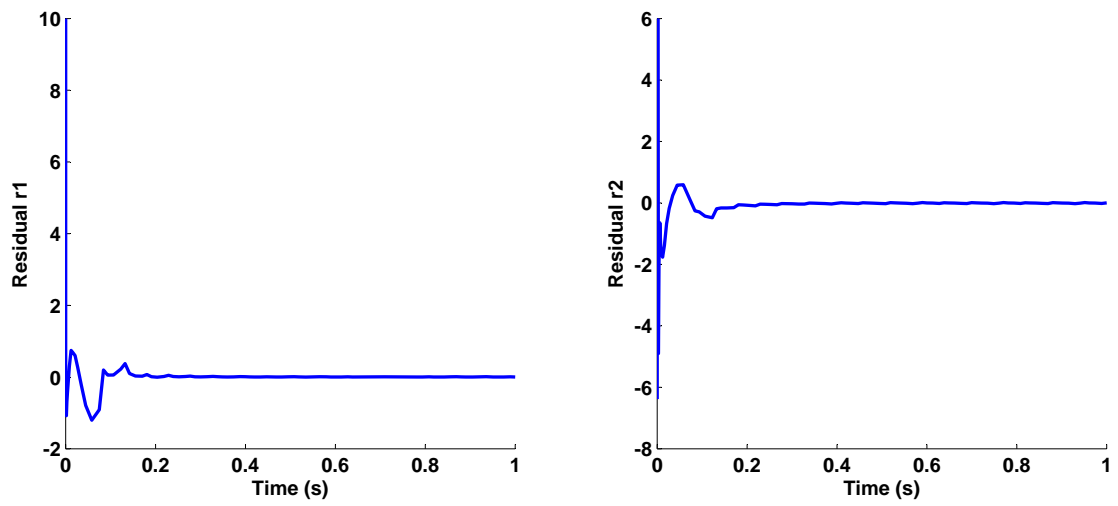

Fig. 14: Time response of the residuals in the absence of failures.

ence of a degradation in the system, represented in this application by a drift in the electrical resistance of the motor's winding, the residuals affected by this drift will respond and leave their nominal values (which were initially close to zero) to move towards other values depending on the magnitude and the form (or trend) of the degradation. The variations of the two residuals due to the motor's winding electrical resistance are shown in figure 15 for a linear degradation and figure 16 for a nonlinear degradation. Also, the change in
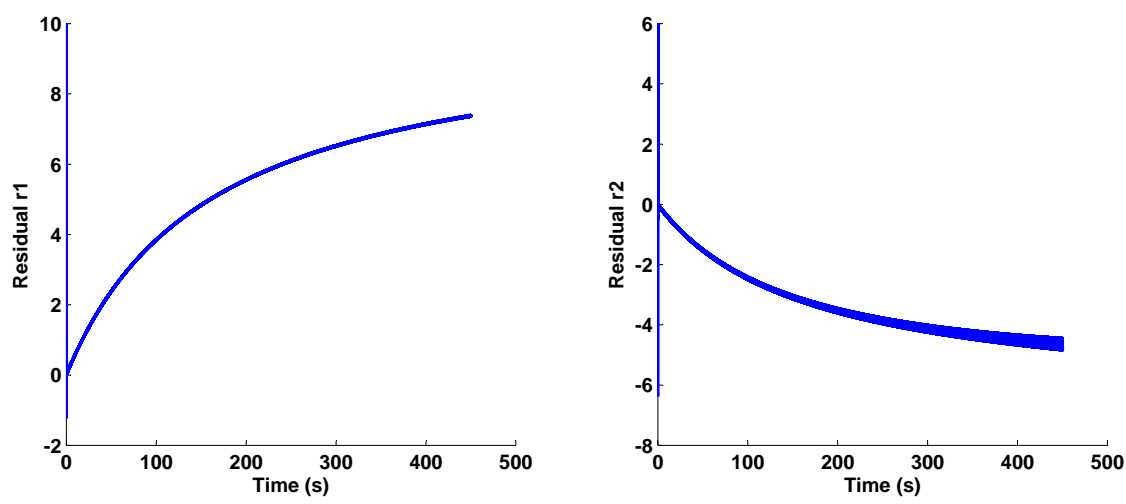

Fig. 15: Time response of the residuals in the presence of a linear degradation. 

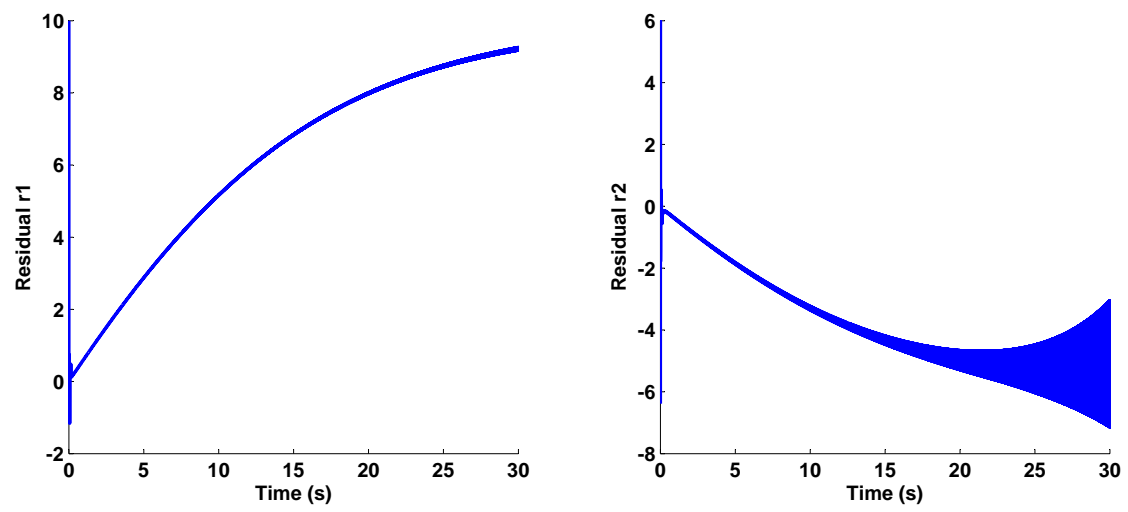

Fig. 16: Time response of the residuals in the presence of a nonlinear degradation.

the system's dynamic can be observed through its time response (figure 17).

The remaining useful life of the mechatronic system can then be calculated
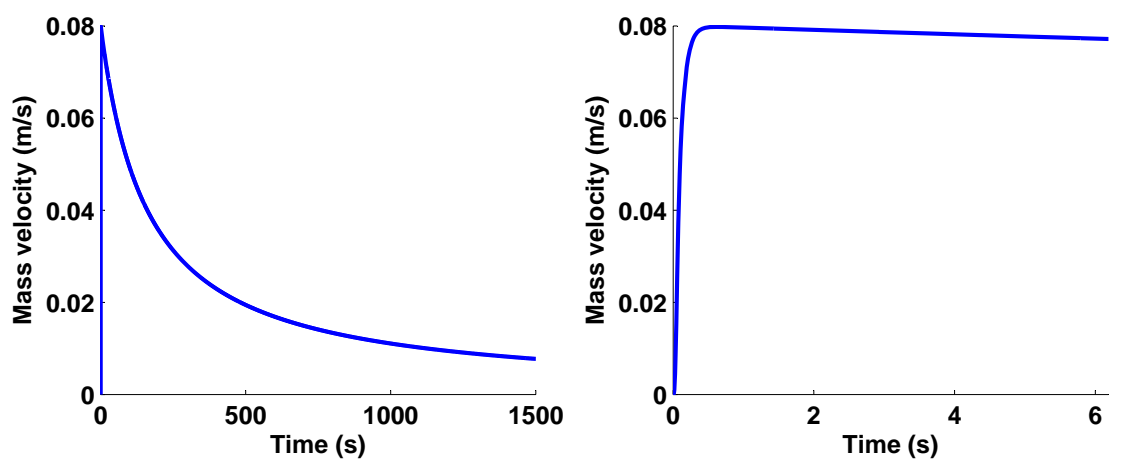

Fig. 17: Time response of the system under a linear degradation (left figure) and zoom on the first seconds (right figure).

according to defined performance criteria (related to the system's precision, time response, stability, etc.). The performance criteria chosen for the RUL calculation can be prioritized: one can imagine that the stability of the system should be more important than its time response. In this application, the system remains stable despite the degradation (this can be verified by calculating its eigenvalues for different values of the resistance $R_{1}$ ). For this reason, the criterion taken into account to calculate the RUL is the final value of the system which can be obtained from its transfer function. This function, called 


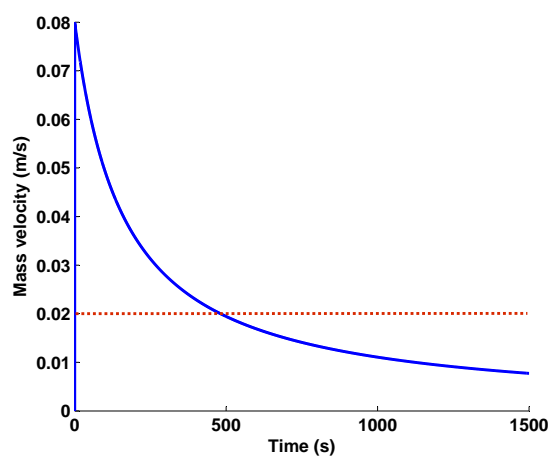

(a)

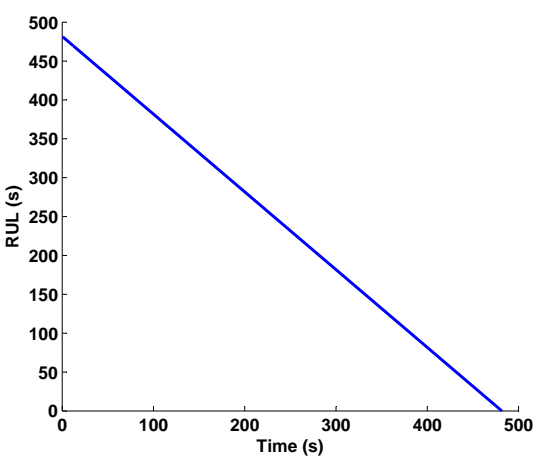

(b)

Fig. 18: Fault threshold value equal to $0.02 \mathrm{~m} / \mathrm{s}$ (a) and estimated RUL (b) for a linear degradation.
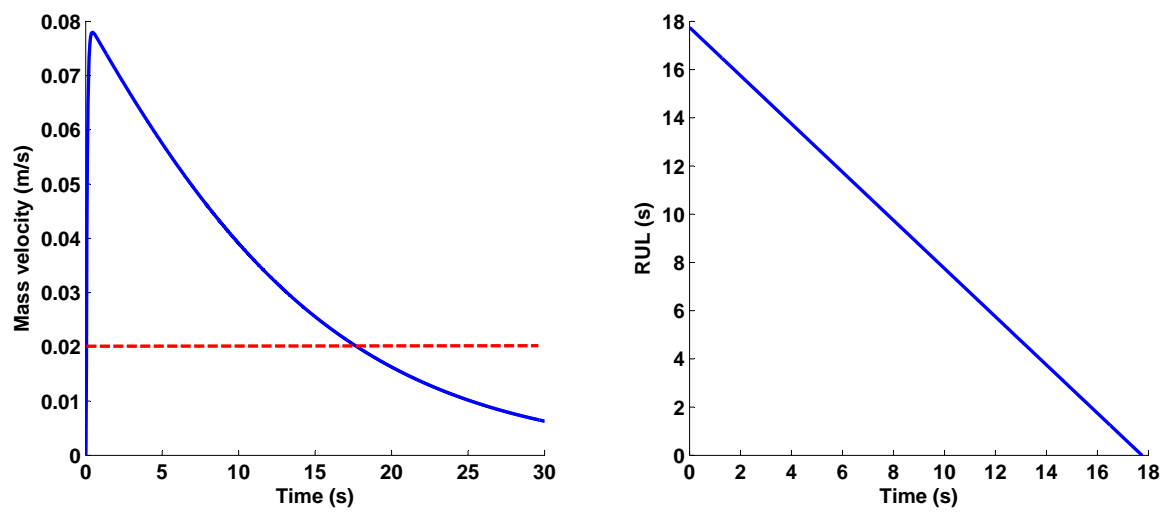

Fig. 19: Fault threshold value equal to $0.02 \mathrm{~m} / \mathrm{s}$ (a) and estimated RUL (b) for a nonlinear degradation.

$H(p)$, can be derived from the state space model given in equation (12):

$$
H(p)=\frac{Y(p)}{E(p)}=C \cdot(p \cdot I-A)^{-1} \cdot B+D
$$

where $E$ is the input of the system, $Y$ is its output (expressed in the Laplace domain), $p$ is the Laplace variable and $A, B, C$, and $D$ are the matrices of the state space model. The steady state of the system for a step input $E(t)$ can then be calculated by using the final value theorem on the transfer function $H(p)$ :

$$
y(t)=\lim _{p \rightarrow 0} p \cdot H(p) \cdot E(p)=\lim _{p \rightarrow 0}\left[C \cdot(p \cdot I-A)^{-1} \cdot B+D\right]=-C \cdot A^{-1} \cdot B
$$

The result of equation (23) shows that the steady state of the system depends on the physical parameters present in the matrices $A, B$ and $C$ (the matrix $D$ 
being equal to zero). Initially and in the absence of the degradation, the steady state value is equal to $0.08 \mathrm{~m} / \mathrm{s}$. However, in the presence of the degradation, the final value changes and decreases to reach a value which is less than 0.01 $\mathrm{m} / \mathrm{s}$. If we set a limit under which the final value is critical (corresponding to failure threshold), the RUL can then be calculated according to the formula given in equation (8). The figures 18 and 19 show the estimated RUL for both linear and nonlinear degradation models and for a steady state threshold equal to $0.02 \mathrm{~m} / \mathrm{s}$.

\section{Conclusion}

A hybrid fault prognostic method applied to mechatronic systems is proposed in this paper. The method is a system-oriented approach, which can be applied on a wide range of multi-physical systems. It relies on two main phases. The first phase concerns the construction of the system's behavior and degradation models and also the definition of the thresholds needed in the calculation of the RUL. The second phase deals with the assessment of the system's health state, the prediction of its future one and the estimation of its RUL. The degradation models are obtained by using the residuals and the whole behavior model (including the degradations) is used to do simulations, predictions and RUL calculation. The RUL is calculated according to a final value of the system considered as its acceptable performance.

The advantage of the method is its deterministic aspect, as the dynamic model is obtained through physical modeling leading to precise RUL. Furthermore, different degradation models (linear and nonlinear) are simulated. The method is applied on a mechatronic system and simulation results are obtained. However, the implementation of the proposed method on data acquired from an experimental platform would allow verifying and validating its effectiveness.

\section{Acknowledgment}

The authors thank the editors and the reviewers for their careful review and feedback which helped to improve the quality of the proposed work.

\section{References}

1. AFNOR: Condition monitoring and diagnostics of machines - prognostics - part 1: General guidelines. NF ISO 13381-1 (2005)

2. Chelidze, D., Cusumano, J.: A dynamical systems approach to failure prognosis. Journal of Vibration and Acoustics 126, 2 - 8 (2004)

3. Dong, M., He, D.: A segmental hidden semi-markov model (hsmm)-based diagnostics and prognostics framework and methodology. Mechanical Systems and Signal Processing 21, 2248-2266 (2007)

4. Heng, A., Tan, A.C., Mathew, J., Montgomery, N., Banjevic, D., Jardine, A.K.: Intelligent condition-based prediction of machinery reliability. Mechanical Systems and Signal Processing 23(5), $1600-1614$ (2009) 
5. Heng, A., Zhang, S., Tan, A.C., Mathew, J.: Rotating machinery prognostics: State of the art, challenges and opportunities. Mechanical Systems and Signal Processing 23(3), $724-739(2009)$

6. Isermann, R.: Supervision: fault-detection and fault-diagnosis methods. an introduction. Control Engineering Practice 5, 639-652 (1997)

7. Jardine, A.K., Lin, D., Banjevic, D.: A review on machinery diagnostics and prognostics implementing condition-based maintenance. Mechanical Systems and Signal Processing $\mathbf{2 0}(7), 1483-1510(2006)$

8. Karnopp, D., Margolis, D., Rosenberg, R.: Systems Dynamics: Modeling and Simulation of Mechatronic Systems, 2nd edn. John Wiley, New York (2006)

9. Kothamasu, R., Huang, S.H., Verduin, W.H.: System health monitoring and prognostics - a review of current paradigms and practices. Int. Journal of Advanced Manufacturing Technology 28, 1012 - 1024 (2006)

10. Lebold, M., Thurston, M.: Open standards for condition-based maintenance and prognostic systems. In: Proc. 5th Maintenance and Reliability Conference (MARCON) (2001)

11. Luo, J., Pattipati, K.R., Qiao, L., Chigusa, S.: Model-based prognostic techniques applied to a suspension system. Transactions on Systems, Man, and Cybernetics 38, 11561168 (2003)

12. Medjaher, K., Tobon-Mejia, D.A., Zerhouni, N.: Remaining useful life estimation of critical components with application to bearings. IEEE Transactions on Reliability $61(2)$, $292-302(2012)$

13. Merzouki, R., Medjaher, K., Djeziri, M.A., Ould-Bouamama, B.: Backlash fault detection in mechatronic system. Mechatronics 17, $299-310$ (2007)

14. B. Ould Bouamama, Medjaher, K., A.K. Samantaray, Staroswiecki, M.: Supervision of an industrial steam generator. part I: Bond graph modelling. Control Engineering Practice 14(1), 71-83 (2006)

15. Peng, Y., Dong, M., Zuo, M.J.: Current status of machine prognostics in conditionbased maintenance: a review. Int. Journal of Advanced Manufacturing Technology 50, $297-313(2010)$

16. A.K. Samantaray, B. Ould Bouamama: Model-Based Process Supervision: A Bond Graph Approach. Springer (2008)

17. Sikorska, J., Hodkiewicz, M., Ma, L.: Prognostic modelling options for remaining useful life estimation by industry. Mechanical Systems and Signal Processing 25, 1803 - 1836 (2011)

18. Tobon-Mejia, D.A., Medjaher, K., Zerhouni, N.: CNC machine tool's wear diagnostic and prognostic by using dynamic bayesian networks. Mechanical Systems and Signal Processing 28, $167-182(2012)$

19. Venkatasubramanian, V.: Prognostic and diagnostic monitoring of complex systems for product lifecycle management: Challenges and opportunities. Computers \& Chemical Engineering 29(6), $1253-1263$ (2005) 